\title{
DISTÂNCIA DA PAPILLA DUODENI HEPATICA A PAPILLA DUODENI PANCREATICA EM BOVINOS MESTIÇOS DE ZEBU
}

(ESTUDO ESTATISTICO SOBRE 324 OBSERVAÇOES) (*) (*)

\author{
(DISTANCE FROM THE PAPILLA DUODENI HEPATICA TO THE PAPILLA \\ DUODENI PANCREATICA IN BRAHMAN CROSSBREDS)
}

(STATISTLCAL STUDY OF 324 OBSERVATIONS)

\author{
Orlando M. Paiva Antônio A. D'Frrico \\ Assistente Veterinário - Tecnologista \\ 2 gráficos no texto
}

Em recente contribuição para o estudo do trato biliar extra-hepático nos bo. vinos, um de nós, (Paiva e Assis Ribeiro - 1948), teve oportunidade de deduzir, estatìsticamente. a distância do Torus pyloricus à Papilla duodeni hepatica, em mestiços de Zełu.

Aventou-se então a hipótese de que a menor distância média entre toro o papila nos mestiços de Zebu (Bos indicus), comparativamente aos dados conhecidos para hovinos de origem européia (Bos taurus) - principal conclusão estabclecida - poderia ser imputada ao menor comprimento do intestino dos primeiros em relaçio ao dos últimos.

Enquanto se reune material adequado à confirmação ou negativa daquela suposiçăo, apresentarcmos c comentaremos os resultados atinentes à determinação da distância mídia, em certímetros, da Papilla duodeni hepatica à Papilla duodeni pancreatica, em bovinos mestiços de Zebu, com idades variáveis de 28 a 51 meses, aproximadamente, on seja, entre a primeira e última mudas, resultados êsses ob. tidos através cstudo estatístico de 324 tratos duodenais. Pretende-se, mediante Este trabalho, confrontando nossos dados com os referentes aos hovinos de raças não zelunías, averiguar eventuais diferenças raciais c, ao mesmo tempo, divulgar dados inéditos, concernentes à anatomia das vias cxcrctoras pancreáticas do mestiço de Zebu.

Apesar do interêsse que o estudo das relações entre os duetos excretores das glândulas hepática e pancréitica proporciona, tanto à Morfologia quanto à $\mathrm{Pa}$.

(*) Nota prívia apresentada ao IV Congresso Brasileiro de Medicinu Veterinária, realizado no Rio de Janciro, de $22-28 / 1 / 1948$.

(*) Comunicado na V! Reuniāo da Sociedude Anatómicn Luso.Hispano-Americana, realizade de 15.18/5/1949, em Lisbor. 
tologia, de modo particular nos animais domésticos, pela variedade de relaçōes verificáveis, o exame da literatura revela a inexistência, pelo menos a nosso co. nhecimento, de pesquisas sistemáticas sôbre o tema em propísito. Dispomos, à exclusão dos números apontados nos trabalhos de Raumanin e Schmotzer (1912), Mane, Foster e Brimhall (1920), e na monografia de Favil.L. (1923), cujos sumários se seguem, ùnicamente dos citados pelos Tratados de Anatomia Vete. rinária ao alcance, os quais, como é oportuno esclarceer, tratam, quando se referem aos hovinos, quase seguramente, dos de origem européia (Bos taurus).

Em contribuição para a anatomia comparativa -... macro e microscópica do divertículo de Vater e das aberturas dos ductos hepático e pancreático, nos animais domésticos (equijnos, bovinos, ovinos, porcinos, caninos, felinos e aves), Baumans e Schmotzer, bascados em pequeno número de observaçōes. relatam, no capítulo destinado as hovinos, an tratar do Ductus pancreaticus mujor (Wir. sungi), considerado cono único ducto pancrédtico dêssess mamíftros, que êle se abre caudalmente ao ducto colédoco, a cerca de $30-10 \mathrm{~cm}$, ou seja, aproximada. mente, a $80-100 \mathrm{~cm}$ dc piloro. Como sucede em relaçāo ao colédoro. continuam os AA., o ducto pancrcático atravessa, obliquamente, a parcde intestinal, percor. rendo.a, todavia, cm menor extensino do que o faz aquêle $(2.3 \mathrm{~cm})$; seu diâmetro alcança $7-8 \mathrm{~mm}$, enquanto, o duodeno, ao nível da abertura do cunducto excretor, atinge cêrca de $3 \mathrm{~cm}$ de largura.

O ducto de Wirsung forma na sua abertura, papila alongada, a Papilla pancréútica, saliente no lume intestinal, tendo $15 \mathrm{~mm}$ de comprimerto, $8.10 \mathrm{~mm}$ de altura c quase o mesmo de largura. A papila, unida a parede to intestino por um dos lados. mostrat a extremidade intciramente livre na extension de $2-3 \mathrm{~mm}$. Não se nota dilatação do ducto de Wirsung à altura da papila.

O segundo pequeno ducto excretor do pancreas, descrito por Mastin e Fravk (in Bacman e Schmotzer), como unindo-se ao ducto colédoco, não fui chcon. trado nos cetsos examinados pelos AA..

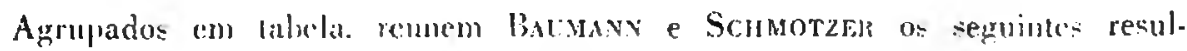
tados, correspondentes is suas oito ohservagües: a) distênciu do pilnto à pa. pila pancréálica: 89.0 - $88.0-97.0-93.0-100.0-84.5-90.0-98.0$ $\mathrm{cm}$, sendo a média de $92.4 \mathrm{~cm} ;$ b) distuncia inter pepilar: $31.5-27.0-35.0$ -.. $39.0-31.0-30.0-38.0-31.0 \mathrm{~cm}$, valores cuja média se fixa em $33.2 \mathrm{~cm}$.

Os índices mídios atinentes: 1) ao diametro do ducto de Wirsung; 2) ao comprimento da papila pancrética; 3) ao diâmetro do intestino, no ponto em

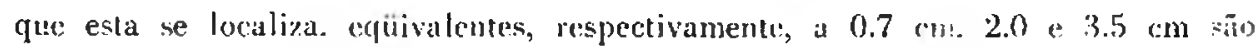
também assinalados. 
Mann, Fuster e Brimhall estudando, comparativamente, a relação entre os ductos biliar e pancreático, em material fresco e fixado, classificam as espécies examinadas $\mathrm{cm}$ três grupos.

No grupo I, o mais simples, enquadram-se mamiferos nos quais ambos os ductos penctram o duodeno separadamente: boi, porco, coclho, cobiajo e "striped gopher" (C. tridemlineatus).

Ao grupo 11, o mais conhecido, pertencem animajs cujos ductos, distintos, apresentam entrada duodenal comum. Regular- ou ocasionaluente, pode-se observar, nêsle grrupo, um segundo ducto pancrético acessório, abrindo-se a alguma distância do orilício cornum aos dois ductos; em alguns casos, o segundo ducto é o maior. A ête grupo filiam-se: homem, macaco ( $M$. rhesus), cão e gato.

Do grupo III fazem parle espécies que mostram o dueto pancreálico desembocando, diretamente, no ducto biliar, a certa distância da abertura dêste no duodeno; são incluídos no grupo: cabra, carnciro, vcado, camondongo, rato e o "pocket gopher" (C. Bursarius).

Represcntando, esquemàticamente, a disposição correspondente a todos os animais grupados, os $\Lambda$. assinalam as distancias do piloro à papila duodenal hepática $(57.5 \mathrm{~cm})$ e à papila duodenal pancreática $(87.5 \mathrm{~cm})$, nos bovinos, sem, contudo, pormenorizar o mincro de observaçôcs.

Na monogralia de Favid.t. sôhre a anatomia do figado dos principais animais domésticos (cavalo, hoi, porco e cão), aprende-se ser único o ducto excreto pancreatico dos bovinos, situando-se o orificio de abertura de 30 a $40 \mathrm{~cm}$ a jusante daquele correspondente ao ducto colédoco.

Moxgakurso (1903) refere apenas que, ordinariamente, os conductos pancreá-

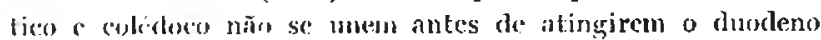

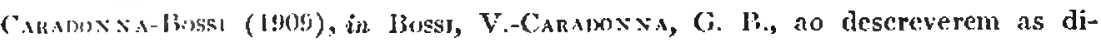
ferencens do princreas nos bovinos, dizem ser ínico seu canal excretor, abrindo-se no

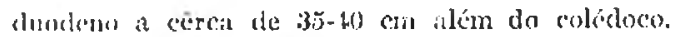

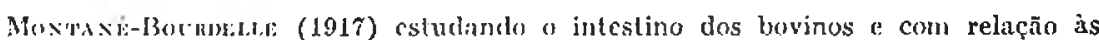
abcrturas dos canais colfiduco e de Wirsung ensinau: estas aberturas são distintas, encontrando-se a primeiri is 60 on $75 \mathrm{~cm}$ a contar do piloro, e a segunda a $35-40$ ou (ii) cri distalmente a esta viltimal

Quanto ao aparclino excretor do páncreas, esclareccm os AA., comporta um

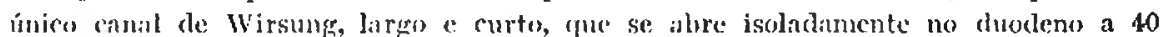
ém, circal, do coléduco (o muito longe do piloro.

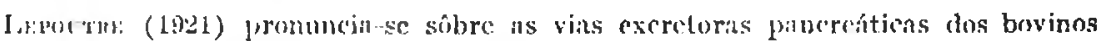

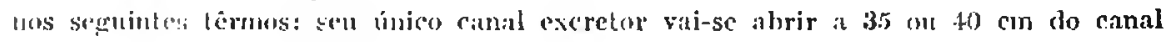
solidoen.

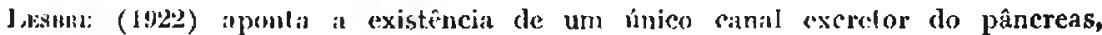
nos borinos, acrescentando: tem 2 a $3 \mathrm{~cm}$ de comprimenlo e 8 a 10 mu de largura, terminitudo it 35 ou $40 \mathrm{~cm}$ distalmente no colédoco. 
Gonzál.s\% y Gancis-AI.Mare\% (1929) afirmam que o pancreas possul uni conducto excretor simples, cuja abcrtura, independente, se encontra no dundeno à distância de 35 a 40 cm para trás da termineçāo do colédoco.

Caradorya (1930), in '/ammerrr, U., expende, no capitulo dedicado ao estudo das diferenģas do pâncrens c com referência aus bovinos, o seguinte patrecrr: scu

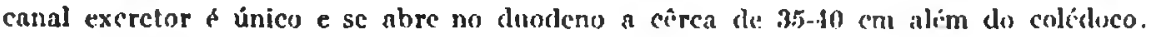

Favilr.r (1931) apresentando as principais diferengas do intestino delyzalo dos ruminantes, cxplica: a abertura única do ducto excretor pancrecitico arhil-se, "w boi, separada da correspondente ao colidoco; situa-se, via de regril, distalmcnte $a$ abertura dêste, isto e, a $30-40 \mathrm{~cm}$ de distînciat.

Ao descrever o páncreas, acentua ninda a existência de un ĺnico canal excretor, que ao invés de abrir-se próxirno do piloro, juntimirnte coin o colíchero, vai desembocar a $50-60 \mathrm{~cm}$ de distância $e$ isoladu.

Elrexurapr-13aum (1932) assinalam a falta do conducto principal do pincreas nos bovinos; quanto a conducto acessúrio viria abrir-se de $30-10 \mathrm{~cm}$ distalmente zo ducto biliar, ou seja de $80-90 \mathrm{~cm}$ (?) ciudalmentc so piloro.

Os mesmos A A., ao apreciar o comportamento do pincreas nos bovinos, confirmam que a abertura do ducto pancreático se localiza de $30-40 \mathrm{~cm}$ Iara trís da abertura do ducto colćdoco, isto $e^{-}$- aqui discordam do que escrevem anteriormente -- de $80-110 \mathrm{~cm}$ de distancia do piloro. O ducto atravessa obliquanente o duodeno, daltura da $4.9 \mathrm{~V}$.I. cếrea de $15 \mathrm{~cm}$ ventralmente a columa vertebral.

Sissurs (1933) e sissor-Grosimas (1915) indicam a posiçato da abertura do con. ducto pancrélices a uns $30 \mathrm{~cm}$ para trás dia desemboradura do conducto biliar, confirmando-o an descrever o pancreas da seguinte mameiral o condurto abandona a

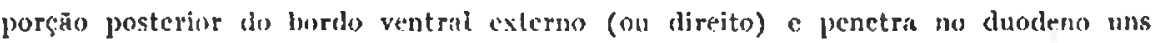
$30 \mathrm{~cm}$ tua is parit trás gue o condurto biliar.

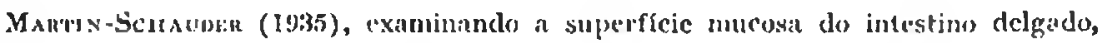
escrevem que os ductos excrefores hepuitico a pancreálico abrem-se, mus bevinus, aquêle a 50-70, iste a $80-110 \mathrm{~cm}$ do piloro.

Con vistas ato pincreas dos ruminantes acrescontan: usualunente existe um unico conducto excretur, orizinitulo da extremidade caudal direitat dit glindulat, que penetra de $80-110 \mathrm{~cm}$ caudalmente a piloro, on seja, a cêrea de $30-10 \mathrm{~cm}$ caudalmente ao ducto colédows. Algumas vizes existe un segundo e curlo comlucto excretor do pânereas, reumido an colidocos.

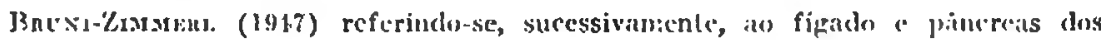
ruminnntes, de modo particular aos seus conductus excretores, anotam: o colídoco

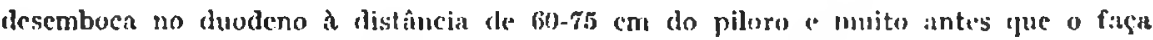
o conducto pincereático; aditando que o paincreas apresenla um único conducto

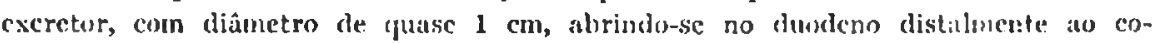
lédoco, de $35-10 \mathrm{~cm}$, portanto, a cèrca da $1 \mathrm{~m}$ do piloru.

Em resumo, nos bovinos, as distâncias apontadas pelas publicacôes especiais e pelos tratadistas citados, para localização da P'upilla duodeni pencreatica, 10. mando-se como ponto de reparo a Papilla duodeni hepaticu, são as seguintes: 
O. M. Paiva e A. A. D'Errico - Papila duodenal pancreática em mest. de Zebu

\begin{tabular}{|c|c|}
\hline BaUMaNiN-ScIIMOTEFr & $33.2 \mathrm{~cm}$ \\
\hline MaNa-Fogter-B Bumirat.l. & 30.0 \\
\hline Favitili & $30-40 \mathrm{~cm}$ \\
\hline Caramoxina-Bosgi (in losgi-Caradonna) & $35-10 \mathrm{~cm}$ \\
\hline Montakt-Botinditid. & $\begin{array}{rc}35-40 & \text { ou } \\
60 & \mathrm{~cm}\end{array}$ \\
\hline Lepotthe & $35-40 \mathrm{~cm}$ \\
\hline$\ldots \ldots \ldots \ldots \ldots$ & $35-40$ cin \\
\hline 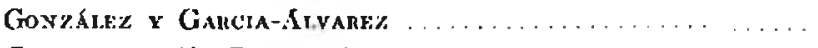 & $35-10 \mathrm{~cm}$ \\
\hline Cahadonat (in ZimmierL) & $35-10 \mathrm{~cm}$ \\
\hline Favilix & $30-10 \mathrm{~cm}$ \\
\hline Friten Detgera-Bauar & $30-10 \mathrm{~cm}$ \\
\hline Sisson c Sisson-Grossman & $30 \mathrm{~cm}$ \\
\hline 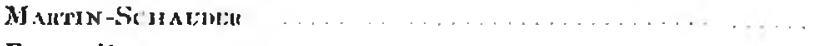 & $30-40 \mathrm{~cm}$ \\
\hline BaUNI-'imgMerL. & $35-40$ \\
\hline
\end{tabular}

A pós o exame dêstes escassos dados bibliográficos, avulta, imediatamente, a inexistência de estudos em basc estatística, de forma a permitir análise comparativa entre os resultados por nós estabelecidos e aquêles apontados pelos diversos AA.. Com efeito, excetuando-se a contribuição de Baumann e Schmotzer, na qual encontramos elementos suficientes para o confronto estatístico, os restantes valores atribuidos à rlistancia interpapilar, embora devan ser considerados como médios, apenas of recem interêsece de conjunto.

Revela-se taminém a oportunidade de discutir, se o único ducto excretor do pâncreas, nos bovinos, corresponde ao Ductus pancreaticus (Wirsungi) ou ao Ducius pancreaticus accessorius (Santorini), respectivamente, Ductus panercaticus ma. jor e Ductus pancreaticks minor. Não se trata à rigor de discutir, jả que a queslío está assentada c'm definitivo. Propomo-nos, tão sòmente, ckamar a atenção para o fato de que, embora em sua quase cotalidade, AA. de trabalhos especializados e tratadistas, concordem em admitir a existência de um único ducto excretor pan. crético, nos bovinos, alguns descrevem-no como sendo o Ductus pancreaticus major (Baymivi o Scinotzer, Montaxí e Bolrdelie), enquanto outros, ou bem especificam tratar-se do Duclus pancreaticus accessorius (F.r.tenirargr e Baum) ou bem deixam de mencionar, como ć o caso da maioria, qual dos dois ductos é o fun. ciona? (Many, Foster e Brimhald, Favilili, Mongripoino, Caradonna e Bossi, Lepoutre, Iezszre, Conzález y Gabch e Alvarez, Caradonna, Sisson, Sisson e (Grossman, Martin e Scinnider, Bruni e Zimmerl).

Particular registro merecem ainda as referências de Martin e Frank (in Bauminy e Scimotzeri e de Martin e Scinuder, com relaģ̃o a um segundo pequeno ducto excretor do pâncreas. unido ao ducto colédoco, presente em alguns casos.

0 desenvolvimento do pâncreas, nos mamíferos - e vem a propósito rememorá-lo para esclarecer as divergências reportadas - se opera a partir de dois 
esboços: dorsal e ventral, sendo êste, inicialmente par (MaURer in HerTwig 1906). Conforme ensina Chinqugi (1940), no Homem, ao mesmo tempo em que se estabelece a fusão dos esboços do pâncreas, ocorre também a reunião dos conductos excretores dêstes esboços, respectivamente, pâncreas dorsal e pâncreas ventral. A seguir nota-se redução do ducto excretor do pâncreas dorsal, enquanto, simultâneamente, o correspondente ao pâncreas ventral aumenta de calibre, vindo a constituir o ducto pancreático principal (Wirsung), cuja ahertura se faz na papila duodenal maior. O ducto excretor do pâncreas dorsal, anastomosado ao ducto principal, passa a constituir o conducto pancreático acessório (Santorini). conservando sua abertura duodenal, isto é, a papila duodenal menor.

Ora, nos bovinos e suînos verifica-se precisamente disposição inversa (AREY - 1936, JoRdan e Kindred - 1942) : enquanto o esbôço pancréntico ventral se sapara, completamente, do ducto colédoco, de modo a não se constiluir um ducto de Wirsung, o eshôço do pâncreas dorsal permanece unido ao intestino, existindo um único ducto excretor, muito amplo, o Ductus Santorini (Sross ...- 1891, P'ensa - 1914), o qual na maioria dos mamíleros, abre-se, no duodeno, caudalmente ao ducto coléduco, ao passo que, no Homem, em tôdas as fases evolutivas nor. mais, termina a nível mais cranial, mais próximo ao piloro (Lokny - 1940). E' bem possivel, todavia, que em alguns casos, segundo acreditamos, possa persistir a relação entre o estôço pancreático ventral e ducto colédoco; só assim, se justificaria a ocorrência do segundo ducto excretor, unido ao colédoco, de que nos falam Martin e Frank, Mlartin e Schaldeb.

Esclarecido o assunto, parecerá estranha e, por isso mesmo, dígna de regis. tro, a anissão em que incorre a maioria dos tratadistas. quando afirma possuir o pâncreas ducto excretor único, scm especificar tralur-se do ducto ancsório (Santorini), muito amplo néstes animais. Assim sendo, deve-se opor absoluta restri-

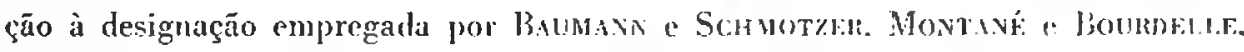
pois, nenhum argumento entriologico a sustenta.

\section{MATERIAT, E METODO}

Para realização da pesquisa utilizamos parte do material recolhido no frigorífico Armour, da Cajilal de Sāo l'aulu, destinado à determinaŗĩo da distância, em centímetros, entre Torus pyloricus e a Papillu duoteni hepaticu e conservado em solução formolada, no Departamento de Anatomid, para futuras investigraçōes. Dos 411 tractos duodenuis de bovinos mestiços do Zebu, emaia examinados, foram considerados $\mathrm{em}$ condiçoes de aproveitamento para o atual extudo, 213, ou seja, 126 de machos, castrados em sua naioria, 9 de fêmeas c 78 pro. venientes de animais cujo sexo não pôde ser averiguado, mas, provàvelmente machos, porquanto, o ahate de fêrneas era raro na ćpoca em que sc reuniu aquêle material de estudo. 
Objetivando a apresentação de resultados o quanto possível completos, decidimos recolher. no mesmo Frigorífico, novo grupo de peças, 125 ao todo, compreendendo 119 de machos e 6 de fêmeas, destinadas ao cálculo da porcentagem de retratilidade, sob a ação do formol, mediante série de medidas efetuadas prévia. e posteriormente à fixaçĩo.

Tal resolução viria, quando menos, permitir-nos conhecer a distância interpapilar em material fresco, fato importantíssimo, já que a previsão de valores. teóricos, a partir da média estabelecida em duodenos fixados e vice-versa, se nos afigurava problemítica, pois, o coeficiente de variaçăo, em têrmos dc porcentagem de retratilidade, mostrara-se bastante elevado, em trabalho anterior, relativo à localizaçióo da papila duodenal hepática.

Dêste novo lote de peças eliminamo: 11. retiradas de machos, por nāo reunirem condições de examc. Iim resumo, contanos com 321 tratos duodenais provenientes de 231 machos, 15 fémeas e de 78 animais, provavelmente machos; dentre êstes, 109 (103 machos e 6 fêmeas), prestaram-se à prova de retratilidade.

Quando da colheita do material buscamos assentar dados relativos à raça, ao sexo e à idade. Selecionamos, assim, de modo exclusivo, tratos duodenais de bovinos mestiços de Zebu, de ambos os sexos, com idades variáveis entre cêrca de 28 e 51 meses, limiles correspondentes à substituição, respectivamente, das pinças e cantos de leite, de acôrdo com at fabola de Chibrfi, P, uva e Vhica (1943), claborada para as raças zebuínas.

Como as carcassas de gado zeluino são fàcilmente reconhecidas c como, de outra parte. o sistema de identificaçäo destas carcassas e iespectivas cabeças, exigido pelo serviço de inspeçăo veteriníria, favorece, sobremodo, a anotação dos mais dados requeridos e sua ulterior concatcnação. a maior dificullade se resume à relirada das peças, efetuada sempre pelo mesmo individuo da equipe empenhada nas várias funçōes, em locais diferentes na sala de matança.

() isolamento dos segmentos duodenais foi realizado mediante técnica descrita e seguida en trabalho precedente: após duas secçöes transversais ppraticallas, a primcira, sôbre a regizio pilórica do abomaso, a scgunda. cêrca de um metro distalmente ao piloro, completava-se a separaçio por meio de corte justa-duodenal do pecfueno omento e mesoduodeno, em tôda a extensão das peças.

Remcliclos ao lalıoratório e depois de desfeitas as pregas scrosas, eram os duodenos abertos ao longo do contôno livre; exposta a mucosa, climinava.se, por moio de jacto de ígual. os resíduos alimentares acaso existentes.

Dispostas a seguir sôhre superficie plana, as peças foram medidas, por um de suos (Dtrrico), com auxílio de rigua, a qual adaptamos duas hastes, paralelas, uma fixa e outra múvel. Ajusıada a primeira à papila duodenal maior, deslocava-se a segunda até a papila duodenal menor e lia.se, diretamente, a dis- 
tância interpapilar. Após três mensurações, deduzia-se a respectiva média aritmética.

Postas a fixar pelo espaço de 72 horas, em solução aquosa de formol a $10 \%$, repetia-se a tomada de medidas, agora distendendo-se e fixando-se, convenientemente, os tratos duodenais.

A análise estatística dos dados compreendeu a determinação da média aritmética, do êrro da média e do "t test"; anexamos tabulagens e gráficos da distribuição por freqüências, correspondentes às peças fixadas e frescas, de forma a permitir-nos conhecer a classe normal ou modal; a norma foi também calculada.

\section{RESULTADOS}

A distância interpapilar, determinada em 324 tratos duodenais fixados, forneceu-nos para a média e respectivo êrro os seguintes índices: $24.7 \pm 0.28 \mathrm{~cm}$. Estes valores foram assinalados meio a amplitude de $34.8 \mathrm{~cm}$, correspondendo os dados máximo e mínimo a 13.1 e $47.9 \mathrm{~cm}$. 0 coeficiente de variabilidade atingiu a $20.6 \%$.

Segundo se esclareceu, reunimos 109 duodenos, destinados ao cálculo da retratilidade, devendo, para tanto, ser submetidos à mensuração, antes e depois de fixados. A média e êrro da média registrados nêste grupo, prèviamente à fixa. ção, alcançaram $27.8 \pm 0.51 \mathrm{~cm}$, sendo a amplitude de variação e os valores li. mites, na ordem enumerada: 25.6 - 18.3 e $43.9 \mathrm{~cm}$. Quanto ao coeficiente de variabilidade ascendeu a $19 \%$.

O mesmo material, após a fixação, permiliu estabelecer, para a distância entre os ductos colćdoco e pancreálico, as seguintes características estalísticas: $24.8 \pm 0.47 \mathrm{~cm}$. A amplitude de variação, equiivalente a $22.3 \mathrm{~cm}$, teve por limites 16.6 e $38.9 \mathrm{~cm}$, enquanto, o coeficiente de variabilidade, foi encontrado igual a $19.7 \%$.

A diferença de $3 \mathrm{~cm}$ exatos. entre as distâncias médias interpapilares, calcula. da no grupo de 109 tratos duodenais, antes $(27.8 \mathrm{~cm})$ e após a fixação (24.8 $\mathrm{em})$, é destituida de major interêsss: prático, embora, através a aplicação do "t test", se revele, estatisticamente significante.

Como se havia previsto, a dedução do coeficiente de variabilidade, a partir da porcentagem média de retratilidade, nas 109 peças, permitiu inferíssemos, por bastante clevado ( $52 \%)$, ser irrealizível a previsão de dados teóricos para peças frescas. tomando por base números experimentais determinados em material fixado. A porcentagem média de retratilidade e seu desvio padrāo clevaram-se a $10.52 \pm 5.12 \%$; por outras palavras, confirmando quanto se disse a propósito, ao estabelecer a distîncia do toro pilórico à abertura do ducto colédoco, os resultados assentados $\mathrm{cm}$ material fresco representam, com maior exatidão, a con- 
dição anatômica média, relativa à distância que separa as papilas duodenais hepática e pancreática.

A tabulagem dos valores assinalados nas 324 peças fixadas (Quadro I), correspondente à distribuição por freqüênucias, em classes de $2 \mathrm{~cm}$ de intervalo, com indicaçäo das respectivas porcentagens, demionstra que a classe de $24-26 \mathrm{~cm}$, com 65 observaçües, ou scja, $20.06 \%$ do total, é a mais densa em valores; a moda dessa distribuição eqüivale a $24.85 \mathrm{~cm}$.

QUADRO I

Distribuição global dos valores referentes às 324 peças fixadas.

\begin{tabular}{c|c|c}
\hline Classes (cm cm) & N.o de observaçöes & $\%$ \\
\hline $12-14$ & 1.0 & 0.31 \\
$14-16$ & 4.5 & 1.39 \\
$16-18$ & 14.5 & 4.47 \\
$18-20$ & 38.5 & 11.90 \\
$20-22$ & 49.0 & 15.12 \\
$22-24$ & 42.5 & 13.11 \\
$24-26$ & 65.0 & 20.06 \\
$26-28$ & 31.5 & 9.72 \\
$20-30$ & 29.0 & 8.95 \\
$30-32$ & 23.0 & $\mathbf{7 . 1 0}$ \\
$32-34$ & 9.5 & 2.93 \\
$34-36$ & 7.0 & 2.16 \\
$36-38$ & 5.0 & 1.54 \\
$38-40$ & 2.0 & 0.62 \\
$40-42$ & 1.0 & 0.31 \\
$42-44$ & - & - \\
$44-46$ & - & -31 \\
$46-48$ & 1.0 & 100.00 \\
\hline
\end{tabular}

No Gráfico I acha-se representada a mesma distribuição por freqüências, tendo-se também inscrito a curva normal, a fim de facilitar o confronto dos números atuais com os previsíveis segundo a equaçáo de Gauss-Laplace. 0 desajustamento que se pode observar entre dados experimentais e teúricos, relativos a algumas das ordenadas construídas sôbre os pontos médios de classe, não envolve significaçāo estatística, devendo imputar-se às flutuaçōes de amostras simples, mesmo no caso das ordenadas correspondentes às classes $18-20-24-26$ e $26-28 \mathrm{~cm}$. 


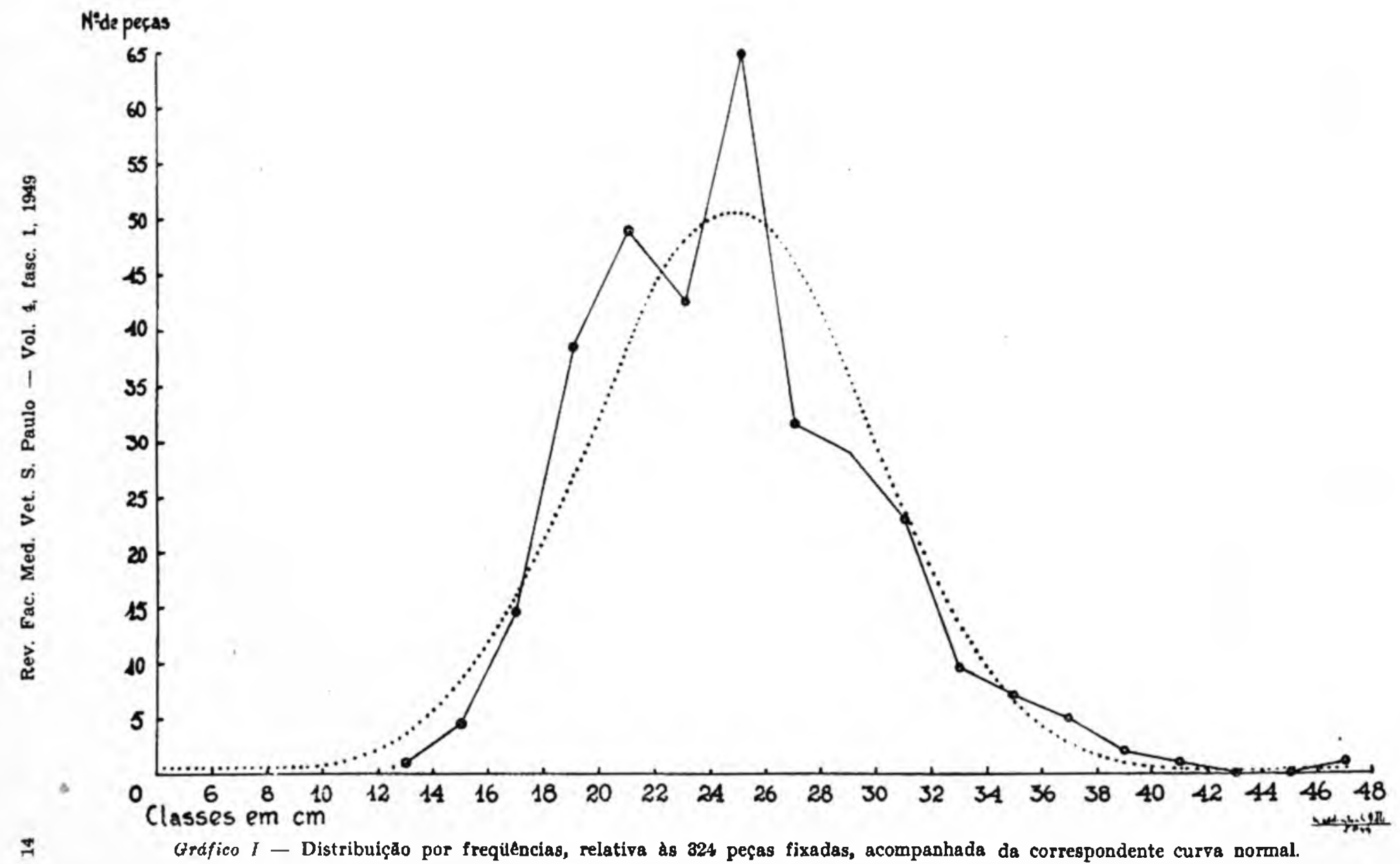


Pode-se ainda aduzir as seguintes notas: a) a média aritmética $(24.7 \mathrm{~cm})$ coincide com a classe modal $(24.26 \mathrm{~cm})$; b) cêrca de $88.9 \%$ dos resultados enquadram-se sta amplitude de $16.34 \mathrm{~cm}$.

Quanto à distribuição por freqüências dos valores obtidos no grupo de 109 duodenos medidos a fresco, cuja tabulagem é indicada no Quadro II, adotando-se o mesmo intervalo de classe e calculadas as respectivas porcentagens, verifica-se que a classe modal é a de $26-28 \mathrm{~cm}$, com 20.5 das variáveis, isto é, $18.8 \%$ do total, sendo a moda igual a $26.97 \mathrm{~cm}$.

\section{QUADRO II}

Distribuiçăo dos dados obtidos, anteriormente à fixaçăo, no grupo de 109 peça

\begin{tabular}{c|c|c}
\hline Classes (em cm) & N.0 de observações & $\%$ \\
\hline $18-20$ & 5.5 & 5.05 \\
$20-22$ & 6.5 & 5.96 \\
$22-24$ & 15.5 & 14.22 \\
$24-26$ & 15.5 & 14.22 \\
$26-28$ & 20.5 & 18.80 \\
$28-30$ & 14.5 & 13.30 \\
$30-32$ & 11.0 & 10.10 \\
$32-34$ & 7.0 & 6.42 \\
$34-36$ & 4.5 & 4.13 \\
$36-38$ & 3.5 & 3.21 \\
$38-40$ & 1.0 & 0.92 \\
$40-42$ & 1.0 & 0.92 \\
$42-44$ & 3.0 & 2.75 \\
& 109.0 & 100.00 \\
\hline
\end{tabular}

Representando no mesmo Gráfico II a distribuição por freqüências atinente ao grupo de 109 peças medidas a fresco, em intervalos de classe com amplitude de $2 \mathrm{~cm}$ e a curva normal correspondente a essa distribuição, nota-se maior a menor coincidência entre as freqüências teóricas e as atuais, cabendo, todavia, qualificar como decorrente da flutuação de amostras, o desajustamento que se nota mesmo nas ordenadas construidas sôbre os pontos médios de classe 23 e $25 \mathrm{~cm}$.

Merecem destaque, ainda, os seguintes fatos: a) a média referente ao grupo em estudo $(27.8 \mathrm{~cm})$ acha-se incluida na classe modal $(26-28 \mathrm{~cm})$; b) aproxinadamente $87.2 \%$ das variáveis situam-se além dos $20 \mathrm{~cm}$ e aquém dos $36 \mathrm{~cm}$. 


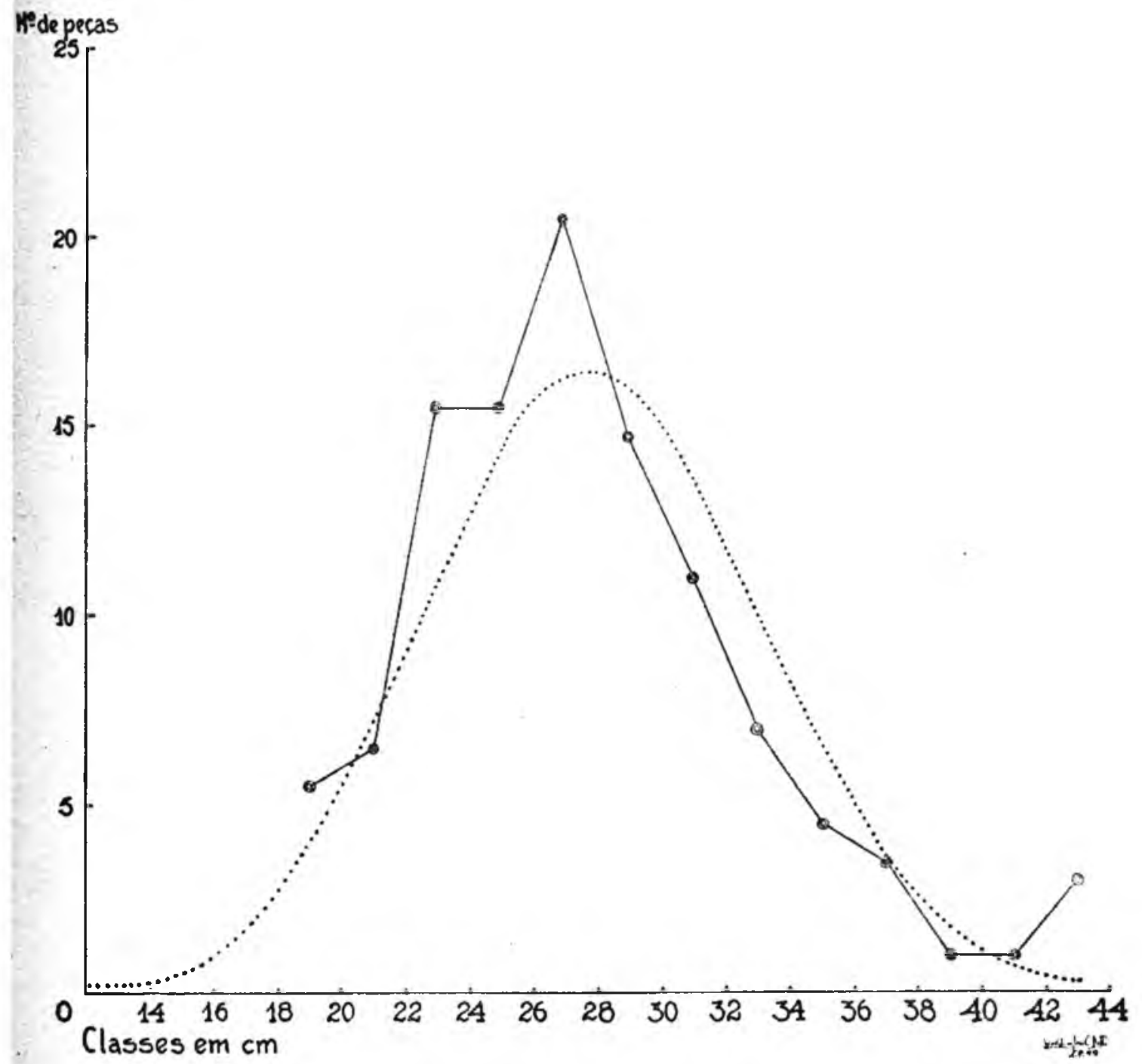

Gráfico 11 - Distribuição por frequiencias, correspondente ao grupo de 109 duodenos não fixados, acompanhada da respectiva curva normal. 
Por fim, no intento de tornar completa a resenha estatística dos valores apurados, damos, simplesmente, a distribuição por freqüências relativa às dimensões registradas nas mesmas 109 peças, posteriormente à fixação, respeitada análoga amplitude de classe (Quadro III).

\section{QUADRO III}

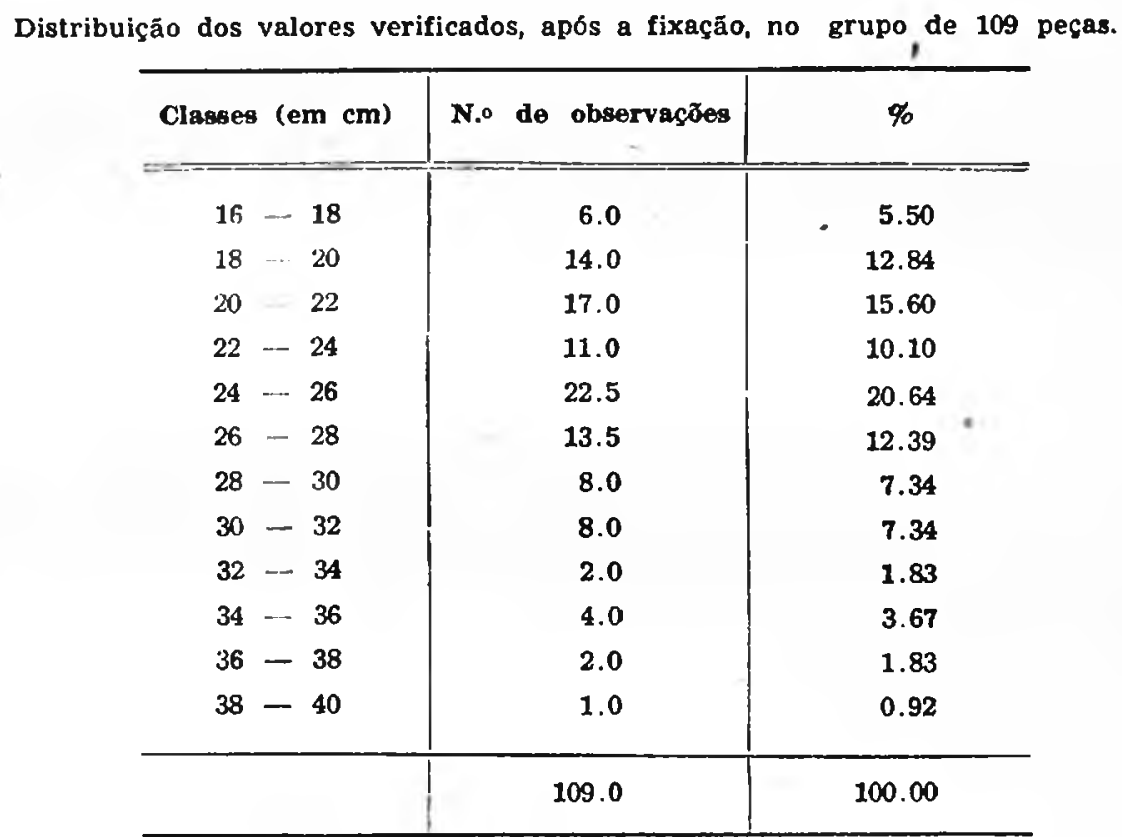

\section{COMENTARIOS}

Passando ao confronto dos resultados apurados, com os dados referidos nos iratados e nas publicaçōes especializadas, repita-se que a análise estatística comparativa, só pode ser efetuada no tocante ao trabalho de Baumann e Schmotzer; os mais valores fornecidos por ManN, Foster e Brimhall, por Favilli e pelos tratadistas, embora a priori, representem a média de certo número de observações, cujo montante é desconhecido, nāo sāo acompanhados dos elementos indispensáveis à aplicação do "t test", para que se julgue da significância das diferenças entre as médias obtidas por nós e pelos diversos AA.. De qualquer forma, nota-se que a distância média interpapilar, no material investigado, tanto em peças frescas $(27.8 \pm 0.51 \mathrm{~cm})$, quanto em peças fixadas $(24.7 \pm 0.28 \mathrm{~cm})$, é sempre inferior à indicada por todos os AA., ainda que escassamente, como sucede em relação a Mann, Foster e Brimhall, Sisson, Sisson e Grossmann $(30.0 \mathrm{~cm})$. Ora, é quase certo - e trata-se de particularidade importantíssima - que as 
peças utilizadas pela totalidade dos AA., para cálculo de tal distância, tênlıam provindo de bovinos de origem européia (Bos tuurus), enquanto, empregou-se no presente estudo, material retirado ùnicamente de mestiços de $Z_{\text {cbu }}$ (Bus indicus).

Quanto a Baumave e Schmotzer, estabeleceram e discriminaram us seguin. tes valores, em oito animais: 1) para a distância entre piloro e papila pancreá. tica: $89.0-88.0-97.0-93.0-100.0-84.5 \ldots 90.0$ p $93.0 \mathrm{~cm}$ sendo a média igual a $92.4 \mathrm{~cm}$; 2) para a distancia interpapilar: $31.5-27.0-35.0$ - 39.0 - 34.0 - 30.0 - 38.0 e $31.0 \mathrm{~cm}$, sendo a média eqüivalente a $33.2 \mathrm{~cm}$. Conhecendo a distância interpapilar e respectiva média, pôde-se calcular c des. vio padrão e o êrro da inédia.

A diferença entre as médias computadas. por nós, em poças näo submetidas à fixação $(27.8 \pm 0.51 \mathrm{~cm})$ e pelos citados AA., em peças fixudas ou pela gli. cerina fenicada e arsênico ou pelo Kayserling $(33.2 \pm 1.4 \mathrm{~cm})$ - processos que não produzem a mesma retratilidade decorrente da ação do formol - atin. gindo a $5.4 \mathrm{~cm}$, conquanto sem maior expressão prática, revela-se. mediante a aplicaçáo do "t test", significante do ponto de vista estatistico. E, frise-se, é das menos clevadas, a média determinada por Bauman e Schmotzrn. presumivelmente, também em hovinos de origem européia.

Estes AA., como se pode obserwar, calcularam igualmente a distância entre - piloro e a papila pancreática, enquanto, nêste trabalho, optou-se pela mensuração apenas do trato interpapilar, pois, dada a natureza do material de estudo, no intúito de afastar as muitas causas de êrro ou, pelo menos, de reduzir.lhe os efeitos - e é êste precisamente o caso - procurou-se trabalhar em tralos duodenais menos longos, recorrendo. para localizaçăo da papila pancreática, ao ponto de reparo mais vizinho.

Como, todavia, faz-se freqüenle menção a tal medida, julgamos interessante apresentar a condição anatômica média a ela referente, em mestiços de Zebu, utilizando, para tanto, os dados de Pava e Assis Ribsiro, concernentes à distância do toro pilórico à papila hepática e os obtidos na presente contribuição, para a distância interpapilar, mediante simples adição dos vialores médios:

\begin{tabular}{l|c|c}
\hline & P. frescas & P. fixadas \\
\hline Dist. do Toro pil. à P.d.h. $\ldots \ldots \ldots \ldots$ & $45.02 \pm 0.31 \mathrm{~cm}$ & $40.41 \pm 0.49 \mathrm{~cm}$ \\
Dist. da P.d.h. à P.d.p. ......... & $27.80 \pm 0.51 \mathrm{~cm}$ & $24.70 \pm 0.28 \mathrm{~cm}$ \\
Dist. do Toro pil. a P.d.p. ........ & $72.82 \mathrm{~cm}$ & $65.11 \mathrm{~cm}$ \\
\hline
\end{tabular}


No momento, mais importante que dar relêvo à discrepância numérica observada para a distância do toro pilórico à papila duodenal pancreática, através cotêjo entre os resultados de Bauman. e Schmotzen $(92.4 \mathrm{~cm})$ e os assinglados no quadro acima, para mestiços de Zebu, quer em pesas frescas $(72.82 \mathrm{~cm})$, quer em peças fixadas $(65.11 \mathrm{~cm})$, é interpretar a já referida significância estatística da diferença média interpapilar, pondo em paralelo valores consignados para hovinos de origem européia e para mestiços de Zebu.

Que fatôres de ordem técnica tais como: retirada, preparo, tratamento e mensuração do material de estudo, possam, eventualmente, acarrețr diferenças significantes sob o ponto de vista estatístico, parece inquestionável. Por isso mesmo, compreender-séí a precaução seguida na determinação do "t test", quando, como se lerí inferido. desprezon-se a condição de material fixado, indicada por BaUmanv e Scrimotzer, para. tomando em consideração simente os processos cmpregados na fixação, confrontá-lo com nosso material fresco. Infelizmentc, a escassez de dados bibliográficos impossibilita se avalie a intervenção de tais fatô. res, mesmo porque, as indicaçōes relativas a método de estudo são quase sempre omissas nêste particular.

O exame dos fatôres de ordem constitucional, por ser muito reduzido o número de fêmeas examinadıs, deve restringir-se à apreciação da idade e raça dos animais utilizados na pesquisa.

Tendo-se ciência de que o intestino delgado dos bovinos atinge suas dimensũes definitivas após os 18 meses, de acôrdo com Auervireimer (in Martin), sòmente foram selecionados espécimes com idades variáveis, aproximadamente, de 28 a 51 mescs. Este critério, além de bastante prático, oferece margem de segurança mais ampla, comparativamente, ao do arrolamento harimétrico, só adotável em lotes submetidos a tratamento homogêneo. Como, entretanto, as publicações consultadas silenciam a propósito do pêso e idade dos exemplares estudados, nada há que acrescentar, restando-nos, pois, discorrer sôbre o fator racial, segundo supomos, fundamento da diferença verificada como estatisticamente significante.

Bi.AcK, Sempl.f e Lusil (1934), cotejando o comprimento do intestino de mestiços de Zebu, meio sangue Zebu-Hereford e Zebu-Shorthorn, com animais puro sangue Hereford e Shorthorn, averiguaram diferenças destituidas de significaçĩo estatística. Em contrapartida, Palva e Assıs Riberro lançaram a hipótese de que a menor distância do toro pilórico à papila duodenal hepática, deveria imputar-se ao menor comprimento do intestino dos bovinos nestiços de Zebu (Bos indicus), comparativamente aos de origem européia (Bos taurus). Com referência à menor distância entre as papilas duodenais hepática e pancreática, nos mestiços 
de Zebu, sugerimos ainda a mesma explicação, apoiando-nos ao fato de têrmos trabalhado, anàlogamente a êstes AA., com espécimes mais de 7/8 e 15/16 de Zebu, circunstância bastante ponderável, a considerar-se o problema genético que suscita.

\section{RESUMO E CONCLUSOES}

No estudo apresentado procurou-se deduzir e analisar, estatisticamente, a distância média da Papilla duodeni hepatica à Papilla duodeni pancreatica, em bovinos mestiços de Zebu, com idades variáveis de 28 (primeira muda) a 51 (úlLima muda) meses, aproximadamente.

O material utilizado abrange 324 peças, assim distribuidas: 231 provenien. tes de machos, na maioria castrados, 15 de fêmeas e as restantes 78 , de animais cujo sexo não se pôde averiguar. Nêste total incluem-se 109 peças 1103 machos e 6 fêmeas), sôbre as quais se efetuaram duas mensuraçōes, antes e após a fixação, no sentido não só de estabelecer a condição anatômica média em tratos duodenais frescos, como também para cálculo da retratilidade, por ação do formol. Outrossim, visava-se indagar a possibilidade de prever valores médios teóricos, para peças frescas, partindo de índices médios apurados em material fixado.

A análise dos valores colhidos compreendeu a determinação da média aritmética, do êrro da média e do "t test" e mais a construção de gráficos da distribuição por freqüências.

Pretendeu-se, mediante esta pesquisa, divulgar dados inéditos, concernentes à anatomia das vias excretoras pancreáticas dos mestiços de Zebu (Bos indicus) e. ao mesmo tempo, confrontando os resultados cam os dados referentes aos hovinos de origem européia (Bos taurus), investigar eventuais diferenças raciais.

0 exame da escassa bibliografia a respeito elucida que, exceptuando-se a contribuição de BaUmanN e SCHMotzer, na qual não são registrados os elementos necessários à análise estatística comparativa, os mais valores atribuidos à distância interpapilar, embora devam ser considerados como médios, não permitem tal cotêjo.

Revela.se, igualmente, através o exame da literatura, a oportunidade de es. clarecer se o ducto excretor do pâncreas, nos bovinos, correspondente ao Ductus pancreaticus (Wirsungi) ou ao Ductus pancreaticus accessorius (Santorini), respectivamente, Ductus pancreaticus major e Ductus pancreaticus minor.

Em sua quase totalidade, AA. de trabalhos especializados e tratadistas, concordam em admitir a existência de um único ducto excretor pancreático; alguns, todavia, descrevem-no como sendo o Ductus pancreaticus major (BaUManN o Schmotzer, Montané e Bourdelle), enquanto outros, ou bem especificam tra- 
tar-se do Ductus pancrealicus accessorius (ELLENBERGER e BaUm) ou bem deixam de mencionar, como é o caso da maioria, qual dos dois ductos é o funcional (Mann, Foster e Brimhall, Favilli, Mongiardino, Caradonna e Bossi, Lepoutre, Lesbre, González y Garcia e Alvarez, Caradonna, Sisson, Sisson e Gross. man, Martin e Scilauder, Bruni e Zimmerl).

O estudo do desenvolvimento do pâncreas, nos mamíferos (SToss, Maurer in Hertwig, Pensa, Arey, Jordan e Kindred), particularmente nos bovinos e nos suinos, aclara que o eshôço pancreático ventral se separa do ducto colédoco, de modo a não se constituir o ducto de Wirsung; por outro lado, o esbôço do pân. creas dorsal permancce unido ao intestino e seu ducto excretor, único e muito amplo nêsses animais, o Ductus Santorini, abre-se, no duodeno, caudalmente à papila duodenal hepática.

Os resultados obtidos sīo os seguintes:

1.") A Papilla duodeni pancreatica, em 324 peças fixadas, achava-se em mćdia a $24.7 \pm 0.28 \mathrm{~cm}$ da Papillo duodeni hepatica, sendo a amplitude da distribuição eqüivalente a $34.8 \mathrm{~cm}$ e os valores mínimo e máximo, respcctivamente, 13.1 e $47.9 \mathrm{~cm}$. O coeficiente de variabilidade atingiu a $20.6 \%$.

2. ) $\Lambda$ média, no grupo de 109 duodenos medidos anteriormente à fixação, foi encontrada igual a 27.8 - $0.51 \mathrm{~cm}$; a amplitude de variação e os valores limites foram, na ordern enumerada: $25.6-13.3-43.9 \mathrm{~cm}$. Quanto ao coeficicnte de variabilidade alcançou $19 \%$.

3. ) O mesmo matcrial, após fixação, ofereceu as seguintes características estatísticas: $2.4 .8 \pm 0.47 \mathrm{~cm}$. A amplitude de variação, igual a $22.3 \mathrm{~cm}$, apresentou por limites 16.6 e $38.9 \mathrm{~cm}$, enquanto, o cocficiente de variabilidade elevou-se a $19.7 \%$.

4..$^{\circ}$ Aplicalo o "t test" para interpretação da diferença, de $3 \mathrm{~cm}$ exatos, entre as midias calculadas no grupo de 109 tratos duodenais, antes $(27.8 \mathrm{~cm})$ e depois $(21.8 \mathrm{~cm})$ da fixação, verifica-se quc, apesar de destituida de maior interêsse prático, deve ser considerada estatisticamente significante.

5. ) Calculada a percentagem de retratilidade no lote de 109 duodenos, revela-se, para a média aritmética e desvio padrão, os seguintes valores: $10.52 \pm$ $\pm 5.42 \%$; o coeficiente de variahilidade, $\mathrm{cm}$ têrmos de percentagem de retração, ascendeu a $52 \%$, evidenciando-se, desta maneira, a impossibilidade de previsáa do valor teórico para a distância interpapilar. caso os 324 tratos duodenais tivessem sirlo medidos prèviamente à fixação. Esta observação torna também recomendável, sejam as medidas tomadas em material fresco, pois, os resultados assim obtidos representam mais exata- e regularmente a condição anatômica média. 
6. ) Sobrepondo às curvas de distribuição por frequêencia as corresponden. tes curvas normais, relativas aos grupos de 324, peças fixadas (Gráfico I) c 109 peças frescas (Cráfico II), possibilita-se o confronto entre os valores experimentais e os respectivos valores teóricos previsíveis. $O$ desajustamento que se pode apreciar com relação a algumas das ordenadas construidas sôbre os pontos médios de classe, não envolve significaçĩo estatística, devendo imputar-se às flutuações de amostras simples.

Com referência ao grupo de 324 peças, acrescente-se que: a) a média aritmética $(24.7 \mathrm{~cm})$ coincide com a classe modal $(24.26 \mathrm{~cm})$; b) a classe modal $(24.26 \mathrm{~cm})$ reune 65 observaçōes, isto é, $20.06 \%$ do total; c) a moda da distribuição eqüivale a $24.85 \mathrm{~cm}$.

Quanto ao grupo de 109 peças frescas, ressalte-se que: a) a média aritmética $(27.8 \mathrm{~cm})$ enquadra-se também na classe modal $(26.28 \mathrm{~cm}) ;$ b) à classe modal $(26.28 \mathrm{~cm})$ correspondem 20.5 das variáveis, ou seja, $18.8 \%$ do tolal; c) a moda da distribuição é igual a $26.97 \mathrm{~cm}$.

$\left.7^{\circ}\right)$ A diferença entre as médias obtidas por nós, em peças não submetidas à fixação $(27.8 \pm 0.51 \mathrm{~cm})$ c por Baumann e Schmotzikr $(33.2 \pm 1.4$ $\mathrm{cm}$ ), provàvelmente em bovinos de origem européia, atinge a $5.1 \mathrm{~cm}$. Conquanto sem maior cxpressão prática, essa diferença, mediante a aplicação do "t test", revela-se estatisticamente significante.

8. $^{\circ}$ Analisando a interferência dos fatôres de ordem técnica e de natureza constitucional na interpretação dos resultados, sugerimos que a menor distância interpapilar estahelecida em bovinos mestiços de Zebu (Bos indicus), comparativamente aos valores atribuidos por tratados e publicargöes especiais para a citada distância, em boviuos de origem européia (Bos taurus), subordina-se. provàvelmente, ao menor comprimento do intestino dos primeiros. A hipótese res. peitante ao menor comprimento do intestino dos mestiços de Zebu, já um de nós (Pave e Assis Ribeino) havia formulado, ao computar a distância do Torus pyloricus à l'apilla duodeni hepatica, nesses animais.

Brack, Skmple: e Lusi, cotejando o comprimento do irtestino de mestiços de Zcbu (meio sangue Zclu-Hereford e meio sangue Zebu-Shorthorn) e de animais puro sangue Hereford e Shorthorn, verificaram diferenças desprovidas de significação estatística, todavia, sustentamos que a menor distância por nós deduzida para a distancia interpapilar possa atribuir-se, justamente, ao menor comprimento do intestino dos mestiços de Zebu, porquanto, os animais com os quais trabalhamos erain mais de $7 / 8$ e $15 / 16$ de Zebu c esta circunstância deve ser levada em grande aprêço, considerando-se o problema genético que envolve. 


\section{SUMMARY AND CONCLUSIONS}

In this study it uas searched and analysed, statistically, thr average distance from the "Papilla duodeni hepatica" to the "I'apilla duodeni pancreatica", on Brahman crossbreds, the age of which varied, approximately, from 28 (change of first incisor) to 51 months (change of fourth incisor).

After detailing the method of study, measures were taken on 324 picces: 231 of males, 15 of female's, the rest proceeding from 78 animals of unknown sex; this total includes 109 duodenal tracts (103 of males and 5 of females) measured before and after fixation, in order to inquire the retractility percentage.

Statistical appreciation comprised the determination of arithmetic mean, standard deviation, standard error of arithmetic mean and t-test; graphics of fre. quency distributions and the correspondent normal curves were also presented.

Both divulge unpublished data on the pancreatic duct of Brahman crossbred and sethle eventual breed differences were the purposes of this investigation.

The bibliography discloses only BAUMANN and ScHmotzER's contribution in which numerical data in relation to the interpapillar distance are computed, thus permitting a comparative statistical study; the remaining isolated values, though average values, can not be subjected to statistical analysis.

The convenionce of making clear whether the pancreatic duct of bovine, single as it is admitted by nearly all the AA., represents the "Ductus pancreaticus" (Wirsungi) or the "Ductus pancreaticus accessorius" (Santorini), seems to be necessary on the ground of discondant AA.'s opinions.

Indeed, Bacmanis and Schmotzer, Montané and Bourdelle describe the pancreatic duct as the "Ductus pancreaticus major"; ElLENBERGen and BAuM as the "Ductus pancrcaticus accessorius"; while the rest (MIANN, Foster and Brimhall, Favili, Monghamino, Caradonna and Bossi, Lepoltre, Lesbre, Gonzílez y Garcia and Álvarez, Cabanovisa, Sisson, Sisson and Grossmann, Martin and Schatnir, BrLin and Zimmar,) omit which the one is the functional.

The development of the pancreas, in mammals (Stoss, Maurel "in" Hert. wic, PeNsa, ARey, Jordan and Kindien), namely in "bovidae" and "suidae". illustrates that, since ventral pancreatic primordial loses its relationship to the common bile duct, the Wirsung's duct is missing; furthermore, as the relationship of dorsal pancreatic primordial and intestine persists, its excretory duct, the wide "Ductus Santorini", opening into the duodenum, caudad to the hepatic duodenal papilla, represents the chicf duct of these animals.

The conclusions listed are the following:

1.") The average distance betueen "Papilla duodeni hepatica" and "Papilla duodeni pancreatica", in 324 fixed pieces, was $24.7 \pm 0.28 \mathrm{~cm}$; the range of the 
data was $34.8 \mathrm{~cm}$ and the lowest and highest values, respectively, 13.1 and 47.9 $\mathrm{cm}$. The coefficient of variation attained $20.6 \%$.

$\left.2 .^{\circ}\right)$ The mean, in the group of 109 fresh duodenal tracts was $27.8 \pm 0.51$ $\mathrm{cm}$; the range and extremes were $25.6 \mathrm{~cm}$ and $18.3-43.9 \mathrm{~cm}$. The coefficient of variation reached $19 \%$.

3.') The same group of pieces, after fixation, offered the following sta. tistical characteristics: arithmetic mean, $24.8 \pm 0.47 \mathrm{~cm}$; range, $22.3 \mathrm{~cm}$; min. imum and maximum items 16.6 and $38.9 \mathrm{~cm}$; coefficient of variation $19.7 \%$.

$\left.4^{\circ}\right)$ The difference of $3 \mathrm{~cm}$ between the two arithmetic means computed (1) this group of 109 duodenal tracts, before $(27.8 \mathrm{~cm})$ and after $(24.8 \mathrm{~cm})$ fi:cution, though of little practical importance, must be considered of statistical significance.

5.') The percentage of retractility determined in the lot of 109 picces, one by one, gives for arithmetic mean and slandard deviation: 10.52 上 $5.42 \%$; the coefficient of variation in terms of percentage of retrectility raised up to $52 \%$. In this manner, it is impossible to obtuin an interpapillur theoretical value for fixed or fresh material sturting from results computed respectively on frish or fixed duodenal tracts. In the same way it secms demonstrated that measures taken on fresh material represent more correctly the average anatomic condition.

6.') Graphics I and II present the frequency distributions referring to the groups of 32.4 (fixed) and 109 (fresh) picces; underlying normal curves represent the theoretical distributions. The discrepuncies can be accepted as due to fluctua. tions of simple sumples.

In the first group: a) the arithmelic mean $(24.7 \mathrm{~cm})$ coincides with the modal class $(24.26 \mathrm{~cm})$; 1) the modal class $(24.26, \mathrm{~cm})$ includes 65.0 items or $20.06 \%$ of all data; c) the mode is equal to $24.35 \mathrm{~cm}$.

In the second one: a) the arithmetic mean $(27.8 \mathrm{~cm})$ also coincides with the modal class $(26.28 \mathrm{~cm})$; b) the modal cless $(26.28 \mathrm{~cm})$ contuins $20 \mathrm{5}$ items or $18.8 \%$ of all dula; c) the mode is equal to $26.97 \mathrm{~cm}$.

7.) The difference of $5.4 \mathrm{~cm}$ between the means calculated by us in fresh material $(27.8 \pm 0.51 \mathrm{~cm})$ and by BAUMAN and ScHMotzer $(3.3 .2 \pm 1.4 \mathrm{~cm})$. probably on European breeds of cattlc, although without practical importance in. volves statistical significance.

8. ) Analysing the interference of technical and constitutional factors in the interpretation of the results it is prosumed that the shorter distance from the hepatic papila to the pancreatic onc in Brahman crossbreds, as compared with non-Brahman cattle, is submitted to the lesser lenght of the intestine in Indian cattle. This hypothesis relative to the lesser lenght of the intestine in Brahman 
crossbreds was already established by PAIVA and AssIS RIBEIRO when studying the distance from "Torus pyloricus" to the "Papilla duodeni hepatica" in such breed of cattle.

Black, Semple and Lush stated differences without statistical significance, on intestine lenght, in a comparative study between Brahman-Herejord, BrahmanShorthorn crossbreds and typical Hereford, Shorthorn cattle; nevertheless, we maintain the supposition referring to the lesser lenght of intestine in Brahman crossbreds as compared with non-Brahman crossbreds, considering that we worked with specimens perhaps more than $7 / 8$ or $15 / 16$ Brahman crossbreds. This circumstance must not be overlooked in view of the genetic problem which is sirred up.

\section{BIBLIOGRAFIA}

Anvr, 1.. B. - 1936 - Developmental Anatomy. 3rd. ed. Philadelphin, W. B. Saunders Company.

Bavana , A. - Schmotzer, B. - 1912 - Beitrügc zur verglcichenden Anatomie des Vater'schen Divertikels un der Mündung der Gallen und Pankrensgänge. österr. Woch. Tierheill., 37(47):169-71; (48):479-81; (49):193-94; (5n):5(36-7; (51):523-4.

Вцаск, W. H. - Simplf, A. T. - Losir, J. L. - 1934 - Beef production and quality as influenced by crossing lBrahman with Hereford and Shorthorn cattle. U.S. Dep. Agrie., Tech. Bull. 417.

Batsi, A.C. - 7immerr, U. - 1917 - Anatomia degli animali domestici 2. Milano, Francesco Vallardi.

Caradonsa, G. B. - 1929 - "in" Zimmer, U. - Trattato di Anatomia Veterinaria 2. Milano, Francesco Vallardi.

Cumanter, G. - 1940 - Trattato di Embriologia 4(2). Milano, Socicta Editrice Libraria.

Cinifri, A. - Pani, O. M. - Velra, J. S. - 1948 - Contribuição para o estudo da cronologia dentíria do Zcbu. Rez. Fne. Med. Vet., S. Paulo, 3(4):251-69.

Em.r.mencer, W. -- Bмem, H. - 1932 - I Iandbuch der verglcichenden Anatomie der Malislicre. 17 Auf. Berlin, Julius Springer.

Favitit, N. - 1923 - l'Anatomia del fegato nei principali animali domestici (Cavallo, bue, maiale, cane). I'isa, 'Tip. A. Cesari.

FAprt.s, N. - 1931 - Nozioni comparale di Anatomia e Fisiologia degli animali rurali. Torimu, tonione lipografico-diditrice Torinese.

González Y Garcin, J. - Sivarey, R. G. - 1929 - Anatomia comparada de los animales domesticos. 3.a cd. Laragoza, "La Académica".

Jornan, H. F. - Kindien, J. F. - 1912 - Text-book of limbryology. 4th. ed. New York, 1). Appleton Century Company.

I:arorot:, 1. - 1921 - Notes du cours d'Anatumie comparée des animnux domestiques. (icmbloux, J. Duculol.

Ls:sme, F. X. .- 1922 -.. I'ricis d'Anatomie compare des animaux domestiques 1. l'aris, J. B, Bailliere et iils.

Innor, C. - Ons, J. - Artino, J. T. de - 1940 - Embriologia humana e comparada (Ontogênese c Teralogênese), 1." ed. S. Paulo, Compatnhia Melhoramentos. 
Mass, F. C. - Fostrik, J. I'. - Bmminal, S. D. - 1920 - The relition of the common bile duct to the pancreatic duct in common domestic and litionatory animals. Jour. Lab. \& Clin. Med., 5(1):203-6.

Martin, P. W. - Scravbra, W. - 1935 - Ielurbuch der Anatomic dev Ilatustiere 3(2). :3 Auf. Stutgrart, Schickhturt \& Ebmer.

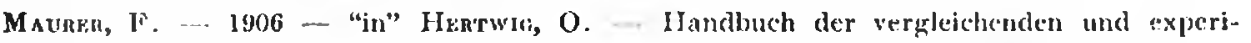
mentellen Lintwickelungslehre der Wirbelticre 2(1). Jena, Ginstav Fischer.

Monanomo, T'. - 1903 - 'Trattato di Anatomia topografica dei nammiferi domestici. Torino, Iuigi Delgrosso.

Montané, L. - Bounuei.le, E. - 1917 -- Anatomie régionale des animaux domestiques 2. Paris, J. B. Baillicre et fils.

PaIva, O. M. - Assis Ribeiro, P. - 1918 - Distância do "Torus pyloricus" à "lapilla duodeni hepatica" em hovinos mestiços de Zelou. Rev. Froc. Med. Vet., S. Laulo, $\mathbf{3}$ (4) :223-46.

Pexan, A. - 1915 - Lo sviluppo del pancreas c delle vie biliari estra-hepatiche in "Bos tanrus". Arch. Ital. Anat. Fmbriol., 13(3):401-51.

Sisson, S. - 1933 - Anatomía de los animales domésticos. Barcelona, Salvat Fiditores, S. A..

Sissox, S. - Grossman, J. D. - 1945 - The Anatomy of domestic animals. 3rd, ed. l'hiladclphia. W. B. Siunders Company.

Strss - 1891 - Zur Lntwickelungsgeschichte des Pankreas. Anat. Anz., 6(23-4):669-9. 